\title{
Expression of HLA-G in patients with B-cell chronic lymphocytic leukemia (B-CLL)
}

\author{
K. Giannopoulos ${ }^{1}$, A. Dmoszyńska², A. Bojarska-Junak ${ }^{1}$, M. Schmitt ${ }^{3}$ and J. Roliński1 ${ }^{1}$ \\ ${ }^{1}$ Department of Clinical Immunology, Medical University, Lublin, Poland; \\ ${ }^{2}$ Department of Heamatooncology Medical University, Lublin, Poland; \\ ${ }^{3}$ Department of Internal Medicine III, University of Ulm, Germany
}

\begin{abstract}
The expression of HLA-G was reported in certain malignancies and its role in escaping from immunosurveillance in cancers was proposed since HLA-G is a nonconventional HLA class I molecule that protects fetus from immunorecognition during pregnancy. Recent studies proposed HLA-G as novel prognostic marker for patients with B-CLL. HLA-G was showed to bear even better prognostic information compared to Zeta-chain associated protein of 70kDa (ZAP-70) and CD38 although some other authors did not find HLA-G expression in CLL. Therefore in this study we characterized the expression of HLA-G on both RNA and protein level. In most of 20 B-CLL patients we were able to detect signal from HLA-G using flow cytometry analysis. The expression of HLA-G was confirmed on messenger level by real-time RT-PCR experiments. No correlation between HLA-G expression and expression of well established prognostic factors such as ZAP-70 and CD38 was detected. These results confirm that HLA-G is expressed on CLL leukemic cells. Furthermore the expression of HLA-G on CLL cells suggests that this molecule might be involved in escaping of CLL cells from immunosurveillance.
\end{abstract}

Key words: human leukocyte antigen-G (HLA-G), B-cell chronic lymphocytic leukemia, B-CLL

\section{Introduction}

B-cell chronic lymphocytic leukemia (B-CLL) is the most frequent malignancy in Western countries [1]. The immunsuppression commonly accompanies other symptoms of disease. This phenomenon might be explained by i) increased level of immunosuppresive cytokine IL-10 [2], ii) increased frequencies of T regulatory cells in B-CLL patients [3,4] iii) decresed function of DC and T cells [5] and iv) hypogammaglobulinemia [6]. However some other mechanisms might be also involved in the escape of CLL cells from immunosurveillance. The expression of HLA-G was reported in certain malignancies and its role in escaping from immunosurveillance in cancers was proposed since HLA-G is a nonconventional HLA class I molecule expressed on trophoblast cells and therefore protecting fetus from immunorecognition during pregnancy [7]. Nuckel et al. [8] reported on the expression of HLA-G on CLL leukemic cells. Moreover it was con-

Correspondence: K. Giannopoulos, Dept. of Clinical

Immunology Medical University of Lublin, Jaczewskiego 8 Str. 20-950 Lublin, Poland; tel.: (+4881) 7425027,

fax: (+4881) 7425027, e-mail: giannop@tlen.pl cluded that HLA-G might constitute a novel prognostic factor superior to well established prognostic factors for patients with B-CLL such as Zeta-chain associated protein of $70 \mathrm{kDa}(\mathrm{ZAP}-70)$ and $\mathrm{CD} 38$. In contrast Polakova et al. [9] in their comprehensive study on HLA-G in hematological malignancies described no expression of this protein on B-CLL cells. Recently, we compared two different staining techniques for the assessment of HLA-G molecule on protein level in B-CLL [10]. Both direct staining as well as non-direct staining were positive for CLL patients however it seemed that primary staining with pure anti-HLA-G antibody followed by staining with secondary antibody was more sensitive. To characterize the expression of HLA-G in B-CLL, in the current study the HLA-G protein was measured using flow cytometry analysis and HLA-G expression was confirmed on messenger RNA level by real-time RT-PCR.

\section{Material and methods}

Patients. Twenty patients with untreated B-CLL, 13 males and 7 females (mean age 65.5 years; range 51-74), were included in this study. Sixteen patients were classified in early stage of disease (stage 0 to 2 according to Rai classification) and four were classi- 
Table 1. Expression of HLA-G in patients with B-CLL (NA-not available).

\begin{tabular}{|c|c|c|c|c|c|c|c|}
\hline Patient & $\Lambda \mathrm{ge}$ & $\begin{array}{l}\text { L.ymph } \\
(\mathrm{G} / \mathrm{L})\end{array}$ & $\begin{array}{l}\text { Stage } \\
\text { (Rai) }\end{array}$ & $\begin{array}{c}\text { CD38 } \\
(\% \mathrm{CD} 5 \mathrm{CD} 19)\end{array}$ & $\begin{array}{c}7 . \wedge \mathrm{P}-70 \\
(\% \mathrm{CD} 5 \mathrm{CD} 19)\end{array}$ & $\begin{array}{c}\text { Hा.A-G } \\
(\% \mathrm{CD} 5 \mathrm{CD} 19)\end{array}$ & $\begin{array}{c}\text { Ratio HL.A-G } \\
\text { /IBP }\end{array}$ \\
\hline 1 & 59 & 20.6 & 1 & 23.45 & 72.06 & 5.27 & 9.87 \\
\hline 2 & 93 & 51.9 & 1 & 3.95 & 45.54 & 15.96 & 52.21 \\
\hline 3 & 61 & 17.6 & 1 & 19.06 & 2.84 & 6.8 & 33.92 \\
\hline 4 & 70 & 170.1 & 4 & 1.95 & 3.19 & 10.53 & 8.26 \\
\hline 5 & 70 & 35.4 & 2 & 2.39 & 1.86 & 8.3 & 23.42 \\
\hline 6 & 57 & 31.9 & 2 & 41.94 & 44.13 & 60.3 & 18.47 \\
\hline 7 & 65 & 14.9 & 0 & 3.23 & 5.77 & 4.46 & 24.16 \\
\hline 8 & 86 & 35.8 & 3 & 6.86 & 24.58 & 7.3 & 370.01 \\
\hline 9 & 46 & 54.1 & 2 & 32.47 & 7.42 & 5.12 & 16.63 \\
\hline 10 & 66 & 9.3 & 1 & 4.13 & 27.56 & 0.35 & 30.11 \\
\hline 11 & 73 & 16.3 & 0 & 11.70 & 12.61 & 10.2 & 11.35 \\
\hline 12 & 57 & 89.7 & 2 & 14.76 & 4.29 & 26.4 & 33.43 \\
\hline 13 & 61 & 73.1 & 4 & 42.86 & 11.54 & 7.02 & 35.48 \\
\hline 14 & 69 & 23.8 & 0 & 1.98 & 21.25 & 14.6 & 25.61 \\
\hline 15 & 52 & 36.9 & 1 & NA & 53.7 & 17.4 & 20.95 \\
\hline 16 & 59 & 30 & 2 & 3.98 & 15.51 & 4.0 & 56.5 \\
\hline 17 & 76 & 58.6 & 2 & 2.86 & 16.89 & 2.0 & 33.33 \\
\hline 18 & 73 & 192.8 & 4 & 93.63 & 12.44 & 14.2 & 21.92 \\
\hline 19 & 66 & 14 & 0 & 1.16 & 10.37 & 2.7 & 33.92 \\
\hline 20 & 51 & 38.5 & 2 & 43.38 & 25.24 & 1.0 & 40.93 \\
\hline
\end{tabular}

fied advanced (stage 3 and 4). The expression of prognostic factors ZAP-70 and CD38 was assessed as described in details [11]. Peripheral blood mononuclear cells (PBMC) were isolated by Ficoll (Biochrom, Germany) density gradient centrifugation. The viability of obtained PBMC was always $>95 \%$, as determined by trypan blue exclusion (Sigma-Aldrich, Germany). The viable cells were quantified in a Neubauer chamber (Zeiss, Germany) and stored for RNA preparation at $-192^{\circ} \mathrm{C}$ in liquid nitrogen.

Flow cytometric analysis of cell surface antigens. The following FITC-, PE- and PerCP-conjugated monoclonal antibodies (moAb) were used for three colour flow cytometry analysis: anti-CD5PE (BD Biosciences,USA), anti-CD19 PerCP (BD Biosciences,USA). For moAb anti-HLA-G pure, clone MEM-G/9 (BD Pharmigen, USA) $10^{6}$ cells were incubated for $20 \mathrm{~min}$. at $4^{\circ} \mathrm{C}$, thereafter washed and stained with secondary goat anti-mouse antibody conjugated with FITC (Dako, Denmark). In the following secondary staining cells were washed twice and stained with CD5 and CD19 for additional 15 minutes. Relevant isotype mouse controls were used. Before flow cytometric analysis, FACScalibur flow cytometer (BD Biosciences, USA) was calibrated. After immunofluorescence staining, cells were washed twice and 20.000 events were acquired and analysed using CellQuest software (BD Biosciences, USA).

mRNA preparation and reverse transcription. For the isolation of mRNA from PBMC, the $\mu$ MACS mRNA Isolation Kit (Miltenyi Biotec, Bergisch Gladbach, Germany) was used according to the manufacturer's instructions. Briefly, after cell lysis, RNA was incubated with $50 \mu$ l Oligo (dT) Micro Beads (Miltenyi Biotec, Bergisch Gladbach, Germany) then isolated in the magnetic field through the MACS column Type $\mu$ (Miltenyi Biotec). After washing isolated mRNA was released from the column by pre-heated Elution Buffer (Miltenyi Biotec). The quality and quantity of the isolated mRNA was assessed using an Eppendorf BioPhotometer (Eppendorf, Hamburg, Germany). Fifty ng of mRNA was reverse transcribed into $20 \mu \mathrm{l}$ of cDNA using a 1st Strand cDNA Synthesis Kit for RT-PCR (AMV) (Roche Diagnostics, Mannheim, Germany). For each RT-PCR $1 \mu \mathrm{l}$ of the cDNA preparation was used.

Quantitative "real time" reverse transcriptase polymerase chain reaction (qRT-PCR). For the quantitative measurement of the mRNA expression of HLA-G real-time RT-PCR was performed using the Light Cycler SYBR Green I technology according to the manufacturer's protocol (Roche Diagnostics). The TATA-Box binding protein (TBP) was used as a house-keeping gene. An initial denaturation step at $95^{\circ} \mathrm{C}$ for 10 min was followed by 40 cycles of $10 \mathrm{~s}$ at $95^{\circ} \mathrm{C}, 15 \mathrm{~s}$ at $62^{\circ} \mathrm{C}, 20 \mathrm{~s}$ at $72^{\circ} \mathrm{C}$ for the TBP. Real-time PCR for HLA-G was carried out with the G.948 forward primer located in exon 5 (5'CTGGTTGTCCTTGCAGCTGTAG-3') and the G.1002 reverse primer located in both sides of exon 5 and exon 6 (5'CCTTTTCAATCTGAGCTCTTCTTTCT-3'). For the gene HLA$\mathrm{G}$ an initial denaturation step at $95^{\circ} \mathrm{C}$ for $10 \mathrm{~min}$ was followed by 45 cycles of $10 \mathrm{~s}$ at $95^{\circ} \mathrm{C}, 10 \mathrm{~s}$ at $62^{\circ} \mathrm{C}, 12 \mathrm{~s}$ at $72^{\circ} \mathrm{C} .0 .1 \mu \mathrm{l}$ of 

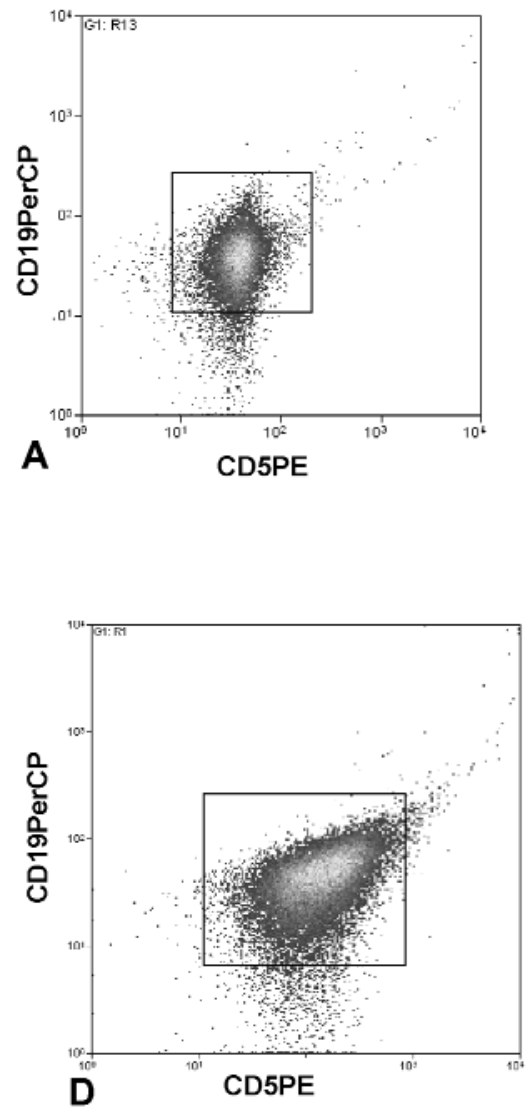
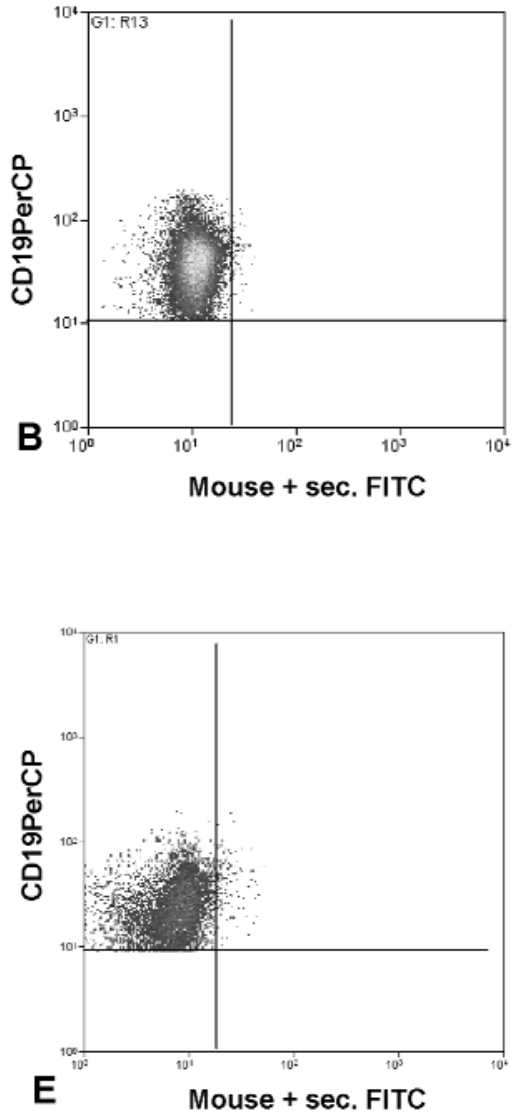
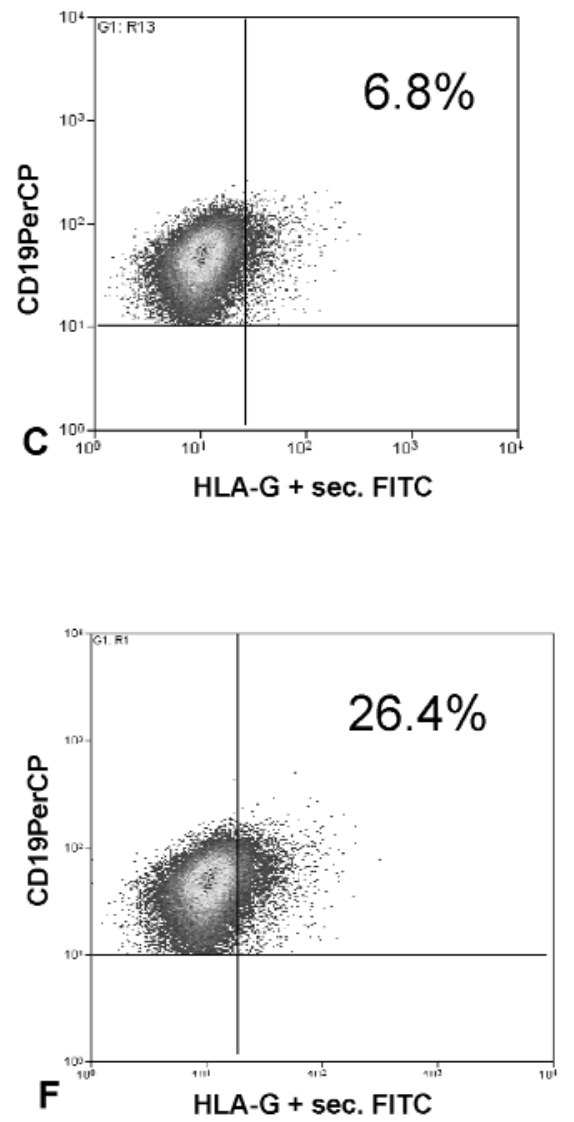

Fig. 1. Assessment of HLA-G expression in chronic lymphocytic leukemia (CLL) cells using flow cytometry. PBMC were analyzed using three color flow cytometry analysis. CLL cells were gated as double positive cells for CD5 and CD19 (A for patient 3 and D for patient 12) and percentage of cell positive for HLA-G were noted (C and F for patients 3 and 12, respectively) with regard to isotype controls $(\mathbf{B}$ and $\mathbf{E})$.

cDNA was used per qRT-PCR. To quantify the mRNA expression of HLA-G, a conventional PCR for HLA-G from placenta was performed and the amount of product cDNA was measured by photometry using an Eppendorf BioPhotometer (Eppendorf). A serial dilution of cDNA was subjected to PCR to obtain standard curves. The amount obtained by quantitative measurement of mRNA in attogram (ag) for HLA-G and TBP was calculated into copy numbers and normalized against the house keeping gene TBP, of which hitherto no retro-pseudogenes are known.

Statistical analysis. To compare HLA-G expression by groups of CLL patients, the non-parametric U Mann-Whitney test was used. Results lower than 0.01 were considered to be significant. Statistical analyses were performed using STATISTICA 5.1 (StatSoft, StatSoft Polska, Poland).

\section{Results}

The expression of HLA-G was detected in all CLL patients both on messenger RNA and protein level.

Using pure moAb, clone MEM-G/9 followed by secondary staining with goat antimouse moAb conjugated with fluorochrome, the median percentage of HLA-G expression in CLL cells was 7.15 (min-max: $0.3-60.3$ ). Examples of flow cytometry analysis are shown in Fig. 1. No correlation with stage was detect- ed. The expression of HLA-G did not differ significantly in patients $\mathrm{CD} 38(+)$ and $\mathrm{CD} 38(-)$ neither in FACS analysis nor in "real time" RT-PCR experiments. No different expression of HLA-G was detected in patients ZAP-70(+) and ZAP-70(-) neither in FACS analysis nor in "real time" RT-PCR experiments. No correlation between mRNA level of HLA-G and HLA-G expression on protein level was found $\left(\mathrm{r}^{2}=-0.28, \mathrm{~ns}\right)$. Results are summarized in Table 1 .

\section{Discussion}

HLA-G is a non-classical MHC molecule expressed in immune-priviledged areas where it plays an important role in the tolerance to fetal antigens. From 1998 when Paul et al. [12] for the first time described the expression of HLA-G in solid tumors, its re-expression during tumorigenesis is linked to suppress tumor recognition by immune system. Enhanced expression of HLA$G$ was found in several malignancies suggesting that HLA-G is involved in the escape of tumor cells from the immunosurveillance [7]. In this study the expression of HLA-G on both messenger and protein level 
was observed. Similar results were shown by Nuckel et al. [8] who found the expression of HLA-G in BCLL on protein level and showed its prognostic properities for patients with B-CLL. In contrast Polakova et al. [9] found no expression of HLA-G on protein level using complex panel of monoclonal antibodies against HLA-G. On the other hand Amiot et al. [12] in comprehensive study on HLA-G expression in lymphoproliferative disorders showed the increased mRNA level of HLA-G. Interestingly enhanced expression of HLA-G mostly resulted in the increased serum levels of soluble HLA-G but hardly with increased expression as HLA molecule on cell surface. Amiot et al. [13] reported on the increased levels of soluble HLA-G (sHLA-G) in B-CLL patients. Augmented concentrations of sHLA-G might favor the progression of cancer by the inhibition of the function of T as well as NK cells [14]. It was suggested that HLA-G inhibits T cells and DC acts via ILT-2 (CD85j) and ILT4 (CD85d) $[15,16]$. CLL cells express the HLA-G receptor ILT-2 [17]. The interaction between HLA-G tetramers with ILT-4 receptor on DC resulted in the reduced expression of HLA-DR [18]. Rebmann et al. [19] speculated whether the reduced expression of HLA-DR on B-CLL cells is attributed to the interaction of sHLA-G with the ILT-2 receptor on B-CLL cells. The discrepancy between the expression of HLA-G on mRNA and protein level observed in this study in CLL cells might indicate that a part of translated HLA-G is released as SHLA-G.

In the current study no correlation of HLA-G expression neither on protein nor on messenger level with well established prognostic factors for B-CLL ZAP-70 and CD38 was detected. Nuckel et al. [8] also didn't find any correlation between HLA-G and ZAP70 and $\mathrm{CD} 38$, however they found that the increased levels of HLA-G (cut-off $23 \%$ ) resulted in a significantly shorter time to progression suggesting that HLA-G might serve as a surrogate prognostic factor in CLL. In our study we could not confirm this relationship, possibly due to a limited number of patients.

In summary, our study revealed the expression of HLA-G in B-CLL on protein level and confirmed using real-time RT-PCR. The expression of HLA-G in B-CLL suggests that this molecule might represent an additional mechanism of tumor escape from the immunosurveillance.

\section{References}

[1] Keating MJ, Chiorazzi N, Messmer B, et al. Biology and treatment of chronic lymphocytic leukemia. Hematology ASH Educ Program. 2003;153-75.

[2] Fayad L, Keating MJ, Reuben JM et al. Interleukin-6 and interleukin-10 levels in chronic lymphocytic leukemia: corre- lation with phenotypic characteristics and outcome. Blood. 2001;97:256-63.

[ 3] Beyer M, Kochanek M, Darabi K et al. Reduced frequencies and suppressive function of $\mathrm{CD} 4+\mathrm{CD} 25$ hi regulatory $\mathrm{T}$ cells in patients with chronic lymphocytic leukemia after therapy with fludarabine. Blood. 2005;106:2018-25.

[4] Giannopoulos K, Schmitt M, Wlasiuk P et al. The high frequency of $\mathrm{T}$ regulatory cells in patients with B-cell chronic lymphocytic leukemia is diminished through treatment with thalidomide. Leukemia. 2008;22:222-4..

[ 5] Orsini E, Guarini A, Chiaretti S, Mauro FR, Foa R. The circulating dendritic cell compartment in patients with chronic lymphocytic leukemia is severely defective and unable to stimulate an effective T-cell response. Cancer Res. 2003;63: 4497-506.

[6] Ravandi F, O'Brien S. Immune defects in patients with chronic lymphocytic leukemia. Cancer Immunol Immunother. 2006;55:197-209.

[ 7] Rouas-Freiss N, Moreau P, Menier C, Carosella ED. HLA-G in cancer: a way to turn off the immune system. Semin Cancer Biol. 2003; 13:325-36.

[ 8] Nuckel H, Rebmann V, Durig J, Duhrsen U, Grosse-Wilde H. HLA-G expression is associated with an unfavorable outcome and immunodeficiency in chronic lymphocytic leukemia. Blood. 2005; 105:1694-8.

[ 9] Polakova K, Krcova M, Kuba D, Russ G. Analysis of HLA-G expression in malignant hematopoetic cells from leukemia patients. Leuk Res. 2003;27:643-8.

[10] Giannopoulos K, Dmoszyńska A, Bojarska-Junak A, Roliński J, Schmitt M. Expression of HLA-G in patients with early stages of B-cell chronic lymphocytic leukemia (B-CLL). Pol J Env Stud. 2006;15:37-40.

[11] Bojarska-Junak A, Rolinski J, Kawiak J. Modification of immunocytochemical ZAP-70 assay for potential clinical application in B-cell chronic lymphocytic leukemia. Folia Histochem Cytobiol. 2005;43:19-23.

[12] Paul P, Rouas-Freiss N, Khalil-Daher I et al. HLA-G expression in melanoma: a way for tumor cells to escape from immunosurveillance. Proc Natl Acad Sci USA. 1998;95 $: 4510-5$.

[13] Amiot L, Le Friec G, Sebti Y et al. HLA-G and lymphoproliferative disorders. Semin Cancer Biol. 2003;13:379-85.

[14] Contini P, Ghio M, Poggi A et al. Soluble HLA-A,-B,-C and $-\mathrm{G}$ molecules induce apoptosis in $\mathrm{T}$ and NK CD8+ cells and inhibit cytotoxic T cell activity through CD8 ligation. Eur $J$ Immunol. 2003;33:125-34.

[15] LeMaoult J, Zafaranloo K, Le Danff C, Carosella ED. HLAG up-regulates ILT2, ILT3, ILT4, and KIR2DL4 in antigen presenting cells, NK cells, and T cells. FASEB J. 2005;19: 662-4.

[16] Liang S, Zhang W, Horuzsko A. Human ILT2 receptor associates with murine MHC class I molecules in vivo and impairs T cell function. Eur J Immunol. 2006;36:2457-71.

[17] Colonna M, Navarro F, Bellón T et al. A common inhibitory receptor for major histocompatibility complex class I molecules on human lymphoid and myelomonocytic cells. $J$ Exp Med. 1997;186:1809-18.

[18] Ristich V, Liang S, Zhang W, Wu J, Horuzsko A. Tolerization of dendritic cells by HLA-G. Eur J Immunol 2005;35:1133-4.

[19] Rebmann V, Nuckel H, Duhrsen U, Grosse-Wilde H. HLA-G in B-chronic lymphocytic leukaemia: Clinical relevance and functional implications. Semin Cancer Biol. 2007;17:430-5.

Submitted: 13 February, 2008 Accepted after reviews: 14 April, 2008 